\title{
Empleabilidad de los graduados de programas de pregrado en odontología de la ciudad de Medellín- Colombia, 2017-2018
}

\author{
Employability of graduates from dental programs \\ in Medellin, Colombia, 2017-2018
}

\section{Empregabilidade de graduados em programas odontológicos em Medellín, Colômbia, 2017-2018}

\section{Ángela María Franco Cortés ${ }^{1}$ Julián Alberto Serna Gómez ${ }^{2}$ Emilia María Ochoa Acosta ${ }^{3}$}

Recibido: 16 de julio de 2019 Aprobado: 12 de noviembre de 2019 Publicado: 20 de diciembre de 2019

Cómo citar este artículo: Franco-Cortés AM, Serna-Gómez JA, Ochoa-Acosta EM. Empleabilidad de los graduados de programas de pregrado en odontología de la ciudad de Medellín-Colombia, 2017-2018. Revista Nac. Odontol. (2019); 15(29), 1-19. doi: https://doi.org/10.16925/2357-4607.2019.02.13

Artículo de investigación. https://doi.org/10.16925/2357-4607.2019.02.13

1 Odontóloga, PhD en Ciencias sociales, niñez y juventud. Profesora titular Facultad de Odontología Universidad de Antioquia. Correo electrónico: angela.franco@udea.edu.co ORCID: https://orcid.org/0000-0001-8579-058X

2 Odontólogo, Magíster en Salud Pública, Profesor Facultad de Odontología Universidad de Antioquia. Correo electrónico: julian.sernag@udea.edu.co ORCID: https://orcid.org/0000-0002-9154-1683

3 Odontóloga, PhD en Ciencias sociales, niñez y juventud. Profesora Facultad de Odontología Universidad de Antioquia. Correo electrónico: Emilia.ochoa7@gmail.com ORCID: https://orcid.org/0000-0001-6765-5712 


\section{Resumen}

Introducción: la empleabilidad se relaciona con la inserción laboral de los graduados según múltiples factores, entre ellos, las condiciones del mercado laboral.

Objetivo: Describir aspectos relacionados con la empleabilidad de los graduados de dos programas de pregrado en Odontología de Medellín, Colombia.

Método: estudio descriptivo en el que participaron 446 odontólogos pertenecientes a dos Universidades de la ciudad, seleccionados mediante muestreo no probabilístico. La información se recogió a través de encuestas y el procesamiento se realizó en el software SPSS versión 22. Se hizo análisis univariado y dos técnicas multivariantes, la técnica del árbol para identificar relaciones estadísticamente significativas entre variables y de correspondencia múltiple, para establecer relaciones entre diversas categorías de variables.

Resultados: el $38 \%$ de los participantes afirmó estar ejerciendo como odontólogo en la modalidad de empleado y el $45.1 \%$ como profesional independiente. El 16,9\% no ejercía su profesión al momento de la encuesta. El promedio salarial fue, en mayor medida, menor a $\$ 1.500 .000$ mensuales (28.8\%). El 32\% de los odontólogos tenía contrato por prestación de servicios y eran, en especial, profesionales con fechas de egreso reciente. El campo laboral de gran parte de los graduados fue la práctica clínica.

Conclusión: algunos aspectos de la empleabilidad que se exploran en el estudio muestran resultados desfavorables para los graduados, entre ellos, la presencia de formas precarias de contratación, el deterioro en los niveles de ingreso, carencia de afiliación al sistema de seguridad social integral y dificultad para insertarse en el mercado laboral, sobre todo para los graduados más recientes.

Palabras clave: empleabilidad, graduados, pregrado, odontología.

\section{Abstract}

Introduction: Employability is related to insertion of graduates into the working market according to a variety of factors, including the conditions of the market itself.

Objective: To describe aspects related to employability of graduates from two undergraduate dental programs in Medellin-Colombia.

Methods: A descriptive study comprising 446 dental professionals, selected by non-probabilistic sampling was carried out. Information was obtained by interviews and processing was performed using software SPSS V 22. A univariate analysis was performed and two multivariate techniques were used: the tree technique to identify statistically significant relations between variables and multiple correspondence to establish relations between different categories of variables.

Results: $38 \%$ of participants stated to be working as employees and $45.1 \%$ as independents. $16.9 \%$ was not working in their profession at the time of the interview. The monthly average salary was mostly under $\$ 1.500 .000$ (28.8\%). $32 \%$ of dental professionals was recently graduated and under services contracts. Most professionals reported to be working in clinical practices.

Conclusion: Some aspects of employability explored in this study showed unfavorable conditions for graduates, including precarious contracts, low income, no affiliation to social security services and difficulty to insert into the working market, especially for recently graduated professionals

Keywords: employability, graduates, undergraduate, dentistry. 


\section{Resumo}

Introdução: a empregabilidade está relacionada à colocação profissional de egressos de acordo com múltiplos fatores, incluindo as condições do mercado de trabalho. Objetivo: Descrever aspectos relacionados à empregabilidade de egressos de dois cursos de graduação em Odontologia em Medellín, Colômbia.

Método: estudo descritivo, no qual participaram 446 dentistas de duas universidades da cidade, selecionados por amostragem não probabilística. As informações foram coletadas através de pesquisas e o processamento foi realizado no software SPSS versão 22. Foram realizadas análises univariadas e duas técnicas multivariadas, a técnica de árvore para identificar relações estatisticamente significativas entre variáveis e correspondência múltipla, para estabelecer relações entre várias categorias de variáveis.

Resultados: $38 \%$ dos participantes afirmaram estar praticando como dentista na modalidade empregado e $45,1 \%$ como profissional independente. $16,9 \%$ não estavam exercendo sua profissão no momento da pesquisa. O salário médio era, em maior medida, inferior a US $\$ 1.500 .000$ por mês $(28,8 \%)$. 32\% dos dentistas tinham contrato para a prestação de serviços e eram, em especial, profissionais com datas recentes de alta. 0 campo de trabalho de muitos dos graduados era a prática clínica.

Conclusão: alguns aspectos da empregabilidade explorados no estudo mostram resultados desfavoráveis para os graduados, entre eles, a presença de formas precárias de contratação, a deterioração dos níveis de renda, a falta de afiliação ao sistema abrangente de seguridade social e a dificuldade ingressar no mercado de trabalho, principalmente para os mais recentes graduados.

Palavras-chave: empregabilidade, graduados, graduação, odontologia.

\section{Introducción}

La empleabilidad es un concepto complejo que hace referencia a la inserción laboral de las personas según distintas variables, relacionadas con las condiciones del contexto social y económico de cada región. Así mismo, se considera que este es un tema que debe estar inmerso en las políticas nacionales, internacionales y entre los objetivos últimos de la educación (1). Su análisis necesita acercarse a los vínculos entre la educación, la fuerza de trabajo y el crecimiento económico, porque el bienestar de la comunidad y la productividad de la sociedad pueden verse afectados cuando existan desequilibrios en el balance entre las organizaciones de servicios, los mismos servicios y los recursos humanos que hacen posible su producción (2). Sin embargo, son escasos los estudios que hayan resumido estos factores en un índice de empleabilidad. La tendencia es incluir solo algunos de los aspectos como las demandas del medio o las tasas de empleo. Otros enfoques, abordan el tema desde la influencia del desarrollo de competencias y de la pertinencia de los currículos (3).

Para el caso de la empleabilidad del talento humano en salud, algunos de los aspectos más relevantes que entran en juego, son los relacionados con las políticas de salud y de educación adoptadas en cada país. Cabe destacar que en Colombia se 
4 Empleabilidad de los graduados de programas de pregrado en odontología de la ciudad de MedellínColombia, 2017-2018

realizó la reforma al sector salud a partir de la entrada en vigencia de la ley 100 de 1993 (4), por la cual se pasó de un modelo de servicios a uno de mercado regulado, perspectiva que determinó nuevas condiciones de integración del recurso humano al mercado laboral. Sin embargo, la reforma en mención no contó con estudios sobre recursos humanos en salud que soportaran sus propósitos y orientaran las decisiones de los agentes de los sectores de educación y salud (5).

Así mismo, el crecimiento en la oferta educativa en salud que ha observado Colombia en los últimos años coincide con la promulgación de la ley 30 de 1992 (6); pues se pasa de 37 instituciones que ofrecen programas de salud en 1984 a 83 en 1999 (7). Para la formación de Odontólogos, en el país existen actualmente 43 programas, implementados por 22 universidades, en 17 ciudades, con cerca de 1900 cupos semestrales (8).

Según datos del Ministerio de Salud de Colombia, las condiciones estructurales en mención han generado consecuencias en las condiciones laborales y en la calidad del empleo de los profesionales de la salud, en especial de los odontólogos. En la actualidad puede afirmarse que hay un desbalance entre la oferta y la integración de odontólogos a la prestación de los servicios de salud en el marco del Sistema General de Seguridad Social de Salud vigente. Este contexto ha sido propicio para el aumento de la oferta laboral de carácter privado, mediante la modalidad de clínicas y figuras asociativas. En este marco han surgido formas de contratación que no se ajustan a la legislación colombiana en materia de trabajo digno (9).

Aunque se han realizado algunos análisis de las condiciones de empleabilidad de los odontólogos de Colombia, es necesario realizar investigaciones periódicamente, con el fin de actualizar los datos y enriquecer el análisis de manera permanente. Según lo expresado, dos programas de odontología en Medellín, realizan este estudio con el objetivo de describir aspectos relacionados con la empleabilidad de sus graduados, incluyendo elementos poco explorados en los estudios realizados como la percepción de los participantes acerca de la formación académica y las diferencias que pueden existir en las condiciones laborales según el sexo.

Se espera, a través de los hallazgos, aportar información para documentar la situación y así participar en la búsqueda de alternativas de mejoramiento de las condiciones de empleabilidad, según las condiciones del mundo laboral actual.

\section{Materiales y métodos}

Se realizó un estudio descriptivo, en el que participaron 446 graduados del programa de odontología de dos instituciones educativas ubicadas en la ciudad de Medellín, la 
Universidad de Antioquia y la Universidad Cooperativa de Colombia, Sede Medellín. Los graduados fueron seleccionados mediante un muestreo no probabilístico. Por el tipo y tamaño de la muestra, este es un estudio exploratorio no concluyente del tema investigado. La información se recolectó mediante una encuesta previamente validada y enviada vía electrónica. El procesamiento de la información se realizó en el software SPSS versión 22. Se realizó un análisis univariado y dos técnicas multivariantes: la técnica del árbol, utilizada para identificar relaciones estadísticamente significativas entre variables dependientes e independientes y de correspondencia múltiple, para identificar relaciones entre diversas categorías de variables, lo cual permitió observar grupos similares en un gráfico estadístico. Se utilizó el método CHAID (detección automática de interacciones mediante chi-cuadrado). Se analizó la relación encontrada entre el tipo de contrato actual del graduado y las siguientes variables independientes: sexo, cobertura y modalidades de afiliación al Sistema General de Seguridad Social en Salud y el rango de ingresos mensuales. La encuesta incluyó algunas preguntas abiertas que se categorizaron según las respuestas obtenidas.

\section{Componente ético}

Se acogieron los principios éticos de la Declaración de Helsinki del 2013 y de la Resolución 8430 de 1993 del Ministerio de Salud de Colombia. Se garantizó la confidencialidad y el anonimato. La participación de los graduados fue voluntaria, se tomó el consentimiento informado y se registró su respectiva firma.

\section{Resultados}

De los 446 graduados, el $91.0 \%$ obtuvo solo título de pregrado en una de las dos universidades participantes, el $8.1 \%$ se graduó del pregrado y el posgrado y el $0.9 \%$ solo de posgrado.

\section{Generalidades de los graduados}

El año de graduación del 58\% de los odontólogos participantes fue entre el 2011 y 2016, seguido por un 26\% entre el 2000 y 2010. Antes del año 2000 se graduó el 16\%.

El 58,3\% residía en Medellín al momento del estudio; el 19,1 \% en otros municipios del área metropolitana; el 10,1\% en otros municipios de Antioquia; el 8,3 en otros departamentos de Colombia; el 2,2\% en Bogotá y el 2\% fuera de Colombia. 
El 68,2\% eran mujeres y el $31.8 \%$ hombres. La edad promedio de los hombres fue de 34,2 (D.E. 10,3), y la de las mujeres fue de 34,0 años (D.E. 11,1).

\section{Aspectos laborales}

Tiempo de primer empleo según sexo. Al 52,7\% de los participantes le tomó menos de seis meses ubicarse laboralmente, como empleado o como independiente. El 8.3\% tardó entre 1 y 2 años, al 1,3\% de los profesionales le tomó más de 2 años conseguir el primer trabajo. No se presentaron diferencias por sexo en el tiempo de espera para ubicarse y aproximadamente el 33,4\% esperó entre seis y doce meses. El $4.3 \%$ de los odontólogos nunca había ejercido su profesión al momento de la encuesta.

Distribución de los graduados según situación laboral y sexo. El 83,1\% ejercía como odontólogo en la modalidad de empleado o como independiente, mientras que el 16,9\% no se desempeñaba como odontólogo al momento de la encuesta. Situación similar en mujeres y hombres. En este resultado se excluye el 4.3\% que nunca había ejercido la profesión.

\section{Aspectos generales de los graduados que no se desempeñaban como odontólogos al momento de la encuesta.}

Tiempo cesante como odontólogo independiente o empleado, según sexo. Se encontraron porcentajes similares en los profesionales que estaban sin ejercer la profesión, entre 6 y 12 meses (22,2\%) y entre uno y dos años (23,6\%). El mayor porcentaje se encontró en aquellos que llevaban menos de seis meses, con un 36,1\%. El 18\% tanto de hombres como de mujeres, no ejercía la profesión hacía más de dos años.

Distribución de las causas para no ejercer la odontología como independiente o empleado, según sexo. La causa principal para no ejercer, bien sea como independiente o empleado, fue que las ofertas no cumplieron con las expectativas, y así lo afirmó el 39,4\% de los encuestados. El 23,9\% mencionó alguna de las siguientes razones para no estar ejerciendo:

- Creación de empresa.

- Ejercicio de otras actividades laborales

- Mejor remuneración en otras áreas.

- Sin ofertas laborales.

- Terminación de contratos. 
El 14,1\% se encontraba cursando posgrado, el 5,6\% se dedicaba a la familia, el $7 \%$ había sido despedido y el 9,9\% fue despedido de su trabajo.

\section{Aspectos generales de los graduados que ejercían la odontología al momento de la encuesta}

El 83,1\% de los profesionales afirmó estar laborando como odontólogo, de ellos el $38 \%$ se desempeñaba como empleado y el $45.1 \%$ como profesional independiente

Tabla 1. Tiempo de duración del empleo, según año de graduación.

\begin{tabular}{|c|c|c|c|c|}
\hline \multirow{2}{*}{$\begin{array}{c}\text { ¿Cuánto hace que tiene el } \\
\text { empleo actual? }\end{array}$} & \multicolumn{3}{|c|}{ Año de Egreso Pregrado } & \multirow{2}{*}{ Total } \\
\hline & Antes del 2000 & 2000 a 2010 & 2011 a 2016 & \\
\hline \multirow{2}{*}{ Menos de 6 meses } & 0 & 0 & 32 & 32 \\
\hline & $0,0 \%$ & $0,0 \%$ & $30.5 \%$ & $19,8 \%$ \\
\hline \multirow{2}{*}{ Entre 6 y 12 meses } & 0 & 3 & 28 & 31 \\
\hline & $0,0 \%$ & $6,8 \%$ & $26,7 \%$ & $19,1 \%$ \\
\hline \multirow{2}{*}{ Entre 1 y 2 años } & 0 & 2 & 26 & 28 \\
\hline & $0,0 \%$ & $4,5 \%$ & $24,8 \%$ & $17,3 \%$ \\
\hline \multirow{2}{*}{ Más de 2 años } & 13 & 39 & 19 & 71 \\
\hline & $100,0 \%$ & $88,6 \%$ & $18,1 \%$ & $43,8 \%$ \\
\hline \multirow{2}{*}{ Total } & 13 & 44 & 105 & 162 \\
\hline & $100,0 \%$ & $100,0 \%$ & $100,0 \%$ & $100,0 \%$ \\
\hline
\end{tabular}

Fuente: elaboración propia.

El 43,8\% de los graduados antes del año 2000 y el 88,6\% del periodo del 2000 al 2010, manifestaron tener su trabajo hacía más de 2 años. Los graduados del 2011 hacia adelante presentaron menor estabilidad, teniendo presente que algunos de ellos eran graduados del 2016.

\section{Tipo de contrato según año de graduación}

El tipo de contratación más frecuente fue a término indefinido $(41,4 \%)$, seguido de contratación a término fijo (26,7\%). Se presentó también la modalidad de trabajo por porcentaje $(21,1 \%)$, y modalidades de contrato por labor terminada $(5,6 \%)$ y prestación de servicios (5,6\%). Los tres últimos tipos de contratación se consideran de prestación de servicios, y en esta modalidad se ubicó el 32\% de los graduados. 
En mayor medida, los contratos a término indefinido los tenían los graduados antes del 2000 (92,3\%), seguidos de los del 2000 al 2010 (47,7\%). Los que se reconocen como trabajadores en modalidades de prestación de servicios, correspondían en mayor porcentaje a personas con fechas de graduación más reciente.

\section{Tipos de institución para la cual trabaja como empleado de la odontología, según año de graduación}

El porcentaje de graduados que labora en instituciones públicas y privadas es similar, cerca al 37\%; sin embargo, se presentan diferencias según año de graduación: más de la mitad (53,8\%) de los graduados de antes del 2000, estaban vinculados a instituciones públicas, y el porcentaje restante a otro tipo de institución.

Del total de los empleados, el 60,5\% manifestó contar con todas las prestaciones sociales, mientras que el 36,4\% afırmó no tener ninguna prestación social. El 3,1\% contaba con prestaciones parciales.

El 66\% de los graduados que estaban empleados contaba con un contrato de tiempo completo, mientras que el 9\% manifestó tener contrato de tiempo parcial, una cuarta parte contaba con contrato de medio tiempo.

El $74,71 \%$ de los empleados realiza actividades clínicas, el $14,71 \%$ se dedica a lo administrativo, a la docencia el 9,41\% y a la investigación el 1,18\%.

Tabla 2. Grado de satisfacción con el contrato según año de graduación.

\begin{tabular}{lcccc}
\hline \multirow{2}{*}{$\begin{array}{c}\text { Satisfacción con el } \\
\text { contrato }\end{array}$} & \multicolumn{3}{c}{ Año de Egreso } & Total \\
\cline { 2 - 4 } & Antes del $\mathbf{2 0 0 0}$ & $\mathbf{2 0 0 0}$ a 2010 & $\mathbf{2 0 1 1}$ a 2016 & \\
\hline \multirow{2}{*}{ Muy baja o baja } & 5 & 26 & 36 & 67 \\
& $35,7 \%$ & $59,1 \%$ & $34,3 \%$ & $41,1 \%$ \\
\hline \multirow{2}{*}{ Mediana } & 2 & 14 & 30 & 46 \\
& $15,4 \%$ & $31,8 \%$ & $28,6 \%$ & $28,4 \%$ \\
\hline \multirow{2}{*}{ Alta o muy alta } & 7 & 4 & 38 & 49 \\
& $50,0 \%$ & $9,1 \%$ & $36,2 \%$ & $30,1 \%$ \\
\hline Total & 14 & 44 & 105 & 162 \\
\hline
\end{tabular}

Fuente: elaboración propia.

El 41,1\% de los graduados que estaban empleados, expresaron tener baja o muy baja satisfacción con el contrato laboral, el 28,4\% afirmó estar medianamente satisfecho y el 31,1\% manifestó tener una satisfacción alta o muy alta con la contratación. 


\section{Argumentos para satisfacción muy baja o baja (respuestas a preguntas abiertas)}

La razón fundamental fue el tipo de contrato, en especial los contratos por prestación de servicios o a término fijo menores a un año.

\section{Argumentos para satisfacción mediana}

Incertidumbre por no tener un salario fijo y depender del flujo de pacientes para alcanzar un salario digno. Hay estabilidad laboral siempre y cuando no se cuestionen las decisiones de las directivas, las ofertas laborales en la ciudad son pocas y en muchos casos mal remuneradas, el empleo está condicionado al cumplimiento de metas por ventas de servicios.

\section{Argumentos para satisfacción muy alta o alta}

Buen salario, horario favorable, contrato a término indefinido, prestaciones sociales, pagos cumplidos.

\section{Percepción sobre su estabilidad laboral como empleado en odontología, según año de graduación}

El 42,3\% percibió como mediana su estabilidad. El 31,9\% correspondió a graduados que consideraron su estabilidad laboral muy baja o baja. Alta la consideró una cuarta parte de los encuestados.

\section{Ingresos y seguridad social de los graduados}

El sexo muestra diferencias en los ingresos mensuales: una tercera parte de las mujeres está en la categoría de menos ingresos, por el 19,3\% de los hombres. Además, entre los que más ingresos poseen, se encuentra el 8,1\% de los hombres y el 4,8\% de las mujeres. En general, los hombres están en las categorías más altas de ingreso. En esta tabla se analizan las respuestas de 427 odontólogos (se incluyen los que están laborando y los que no están laborando. Solo se excluyen los que nunca han ejercido la profesión). 
Colombia, 2017-2018

Tabla 3. Distribución de ingresos, según sexo.

\begin{tabular}{|c|c|c|c|}
\hline \multirow{2}{*}{$\begin{array}{l}\text { Ingresos mensuales } \\
\text { promedio. }\end{array}$} & \multicolumn{2}{|c|}{ Sexo } & \multirow{2}{*}{ Total } \\
\hline & Mujer & Hombre & \\
\hline \multirow{2}{*}{ Menos de 1.500 .000} & 97 & 26 & 123 \\
\hline & $33,2 \%$ & $19,3 \%$ & $28,8 \%$ \\
\hline \multirow{2}{*}{1.500 .000 a 1.999 .999} & 53 & 15 & 68 \\
\hline & $18,2 \%$ & $11,1 \%$ & $15,9 \%$ \\
\hline \multirow{2}{*}{2.000 .000 a 2.999 .999} & 75 & 45 & 120 \\
\hline & $25,7 \%$ & $33,4 \%$ & $27,1 \%$ \\
\hline \multirow{2}{*}{3.000 .000 a 3.999 .999} & 32 & 17 & 49 \\
\hline & $11,0 \%$ & $12,6 \%$ & $11,5 \%$ \\
\hline \multirow{2}{*}{4.000 .000 a 4.999 .999} & 13 & 9 & 22 \\
\hline & $4,5 \%$ & $6,7 \%$ & $5,2 \%$ \\
\hline \multirow{2}{*}{5.000 .000 a 5.999 .999} & 8 & 12 & 20 \\
\hline & $2,7 \%$ & $8,9 \%$ & $4,7 \%$ \\
\hline \multirow{2}{*}{6.000 .0000 más } & 14 & 11 & 25 \\
\hline & $4,8 \%$ & $8,1 \%$ & $5,9 \%$ \\
\hline \multirow{2}{*}{ Total } & 292 & 135 & 427 \\
\hline & $100,0 \%$ & $100,0 \%$ & $100,0 \%$ \\
\hline
\end{tabular}

Fuente: elaboración propia.

En cuanto a la cobertura del Sistema de Seguridad Social, se analizaron las respuestas de los participantes que al momento de la encuesta se desempeñaban como odontólogos, bien como independientes o empleados, es decir, un total de 355 egresados. Los resultados muestran que el mayor porcentaje (67,3\%) está afiliado al Sistema de Seguridad Social Integral; el 19,4\% dice tener parcialmente cubierta la seguridad social y el $13,2 \%$ reconoce no tener ninguna cobertura en el Sistema General de Seguridad Social en Salud. El 87,4\% de los odontólogos, empleados o independientes, están afiliados al régimen contributivo, el 4,8\% pertenece al régimen subsidiado y el 7,8\% no estaba asegurado.

La distribución de opiniones sobre la satisfacción con el ejercicio profesional como empleado o independiente, es relativamente similar, es decir, los porcentajes no difieren mucho en las tres categorías definidas. Sin embargo, la categoría de satisfacción muy baja o baja con el $35,4 \%$ es la más representativa, mientras que los que tienen una satisfacción media, alta o muy alta son porcentajes ligeramente superiores al $30 \%$.

De los que no se encontraban laborando como odontólogos al momento de la encuesta, más de la mitad expresó tener una satisfacción alta o muy alta (54,2\%), 
por el contrario, de los que sí se encontraban laborando como odontólogos, el 40,3\% valoró como baja o muy baja la satisfacción con el ejercicio profesional.

Las tres cuartas partes de los graduados que en algún momento han laborado, consideran que la formación recibida en la universidad, les ha permitido desempeñarse de manera muy adecuada o adecuada (75,9\%), sin embargo, un 15,7\% consideró la formación recibida como mediana para lograr un buen desempeño laboral. Un porcentaje menor, el 8,4\%, expresó que la formación recibida fue baja o muy baja.

\section{Resultados del análisis multivariado}

La variable que presentó mayor relación con la modalidad de trabajo fue la cobertura del Sistema de Seguridad Social Integral. Se aprecia que el 60,3\% de los empleados están totalmente cubiertos, mientras que en los independientes el porcentaje fue del $39,7 \%$. De los participantes que afirmaron contar con cobertura parcial o no contar con ninguna, el 84,5\% laboraba como independiente y el 15,5\% como empleado.

Además, se estableció que dentro de los que estaban cubiertos completamente por el Sistema General de Seguridad Social Integral, se presentaron diferencias significativas por sexo, representadas en un mayor porcentaje de hombres empleados $(72,2 \%)$, mientras en las mujeres los porcentajes son similares entre las que se desempeñan como independientes o como empleadas.

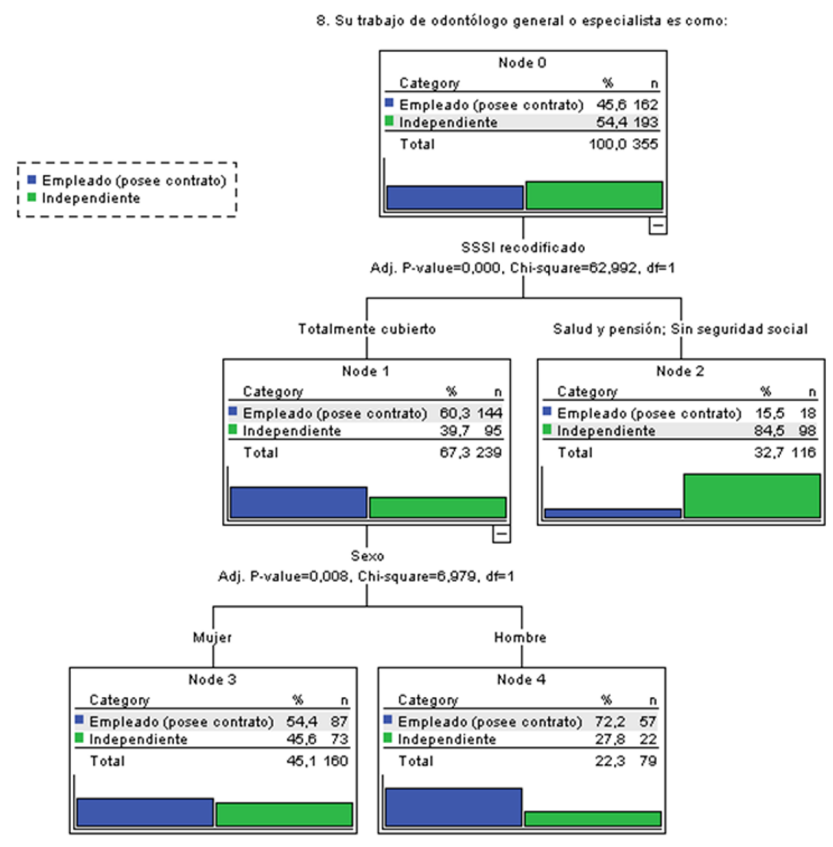

Figura 1. Relación tipo de empleo-variables de seguridad social e ingresos.

Fuente: elaboración propia. 
Contar con contrato o ejercer de forma independiente, presentó relación directa con respecto al año de graduación. Los graduados antes del 2000 y los que solo son graduados de posgrado, son en su mayoría independientes (79,0\%), en cuanto a los graduados del 2000 al 2016 se tienen porcentajes similares (50,0\%).

8. Su trabajo de odontólogo general o especialista es como:

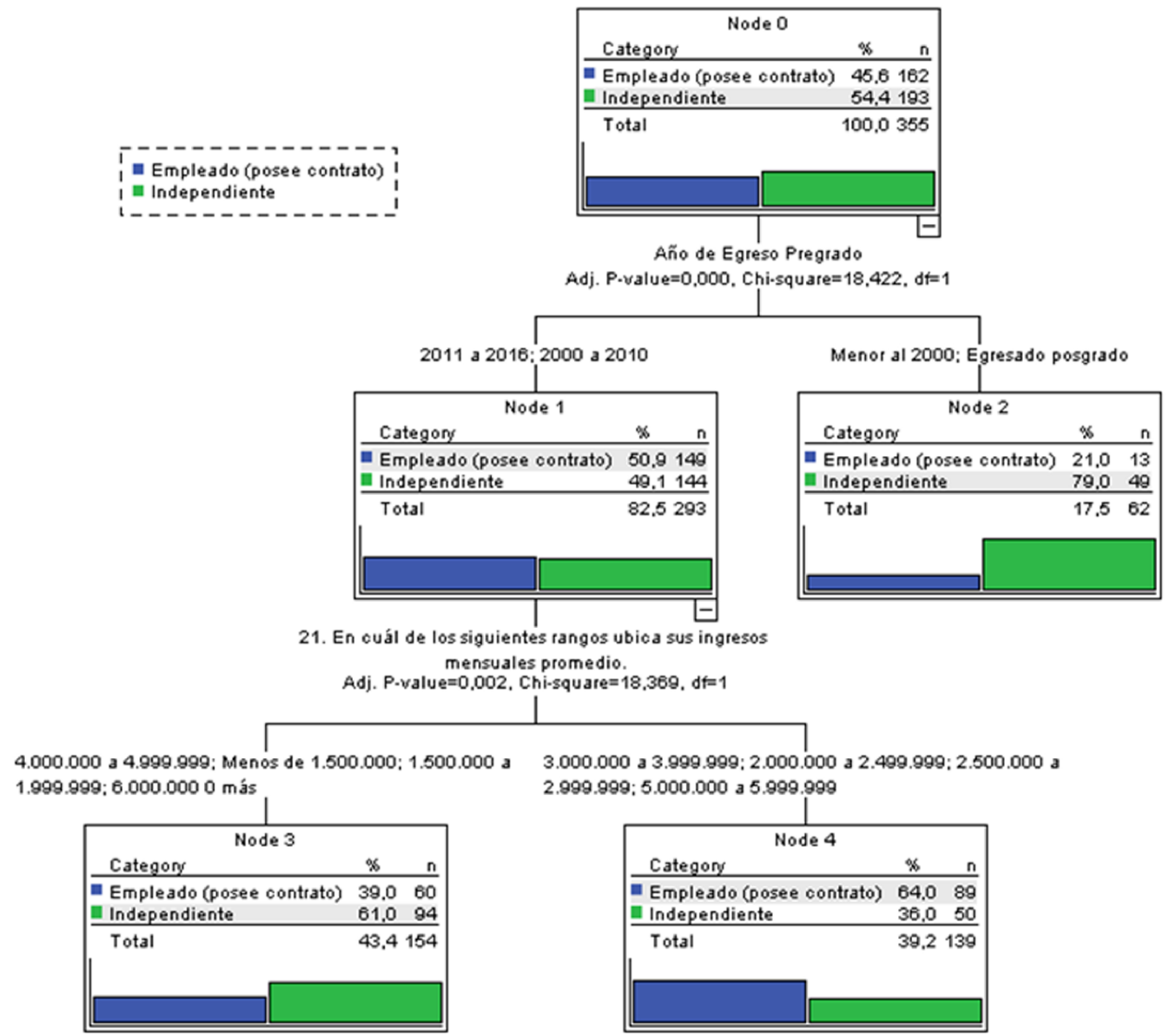

Figura 2. Relación tipo de empleo, año de graduación e ingresos.

Fuente: elaboración propia.

\section{Técnica de Correspondencia Múltiple}

Se detectan dos grupos que permiten identificar algunas características de los hombres y de las mujeres. En uno de ellos se observa cómo las mujeres graduadas entre los años 2011 y 2016, que laboran como independientes, están cerca de los salarios más bajos, ubicados en el rango de menos de \$1.500.000 a \$2.000.000. Los hombres, graduados entre el 2000 y el 2010 se ubican en mayor medida en la modalidad de empleados y perciben salarios entre $\$ 2.000 .000$ y $\$ 4.000 .000$ 


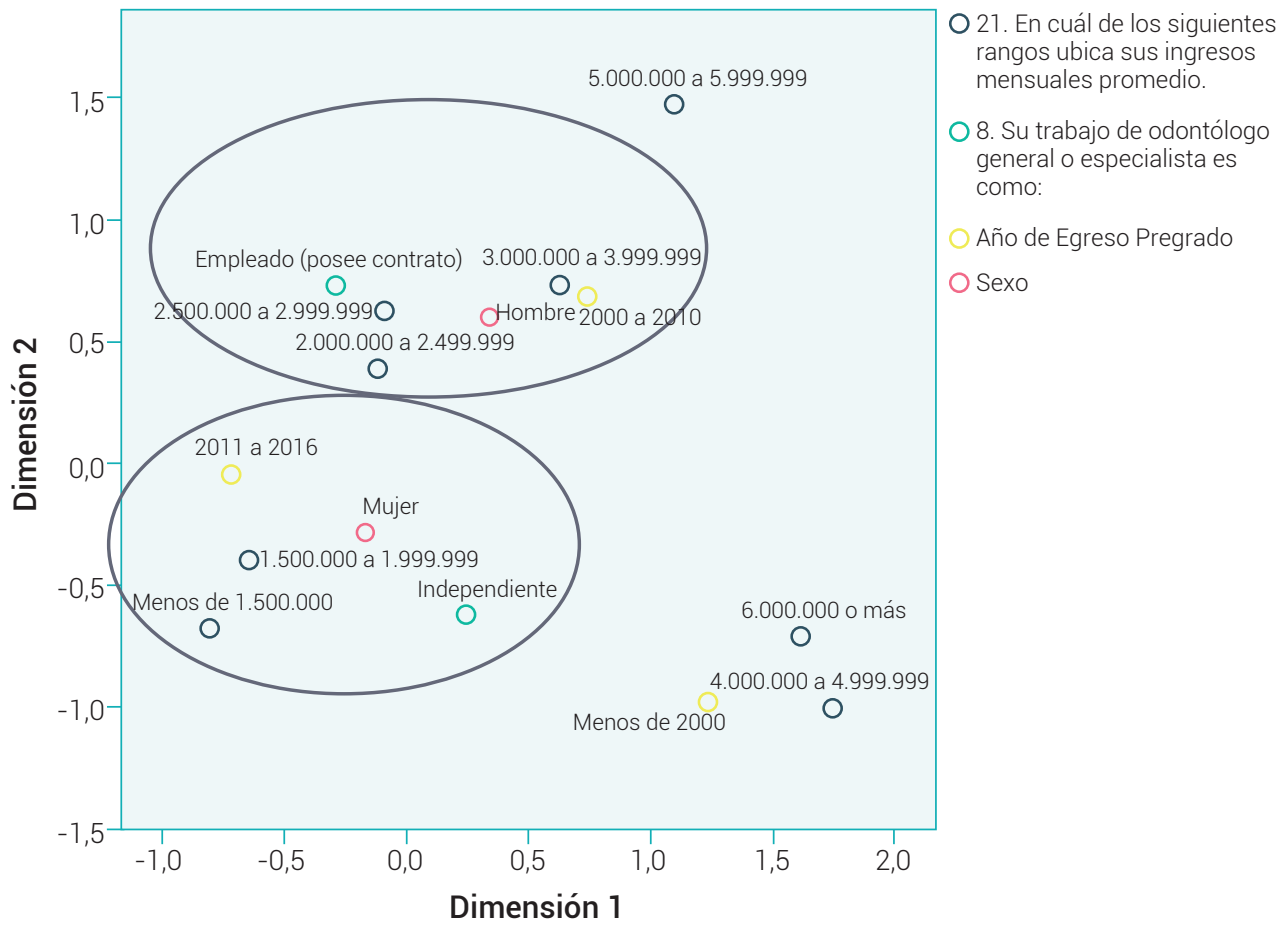

Variable Principal Normalization

Figura 3. Mapa perceptual de las variables tipo de empleo, año de graduación, sexo e ingresos mensuales.

Fuente: elaboración propia.

\section{Discusión}

Los resultados del estudio brindan elementos clave para enriquecer el análisis alrededor del tema de empleabilidad de los graduados del pregrado en Odontología de dos Universidades de Medellín. Lo anterior, teniendo presente que dadas las limitaciones para abarcar el concepto, se muestra el comportamiento de algunas de las variables, no de la totalidad de las que se pueden incluir en los análisis de este fenómeno, porque hay diferencias en la definición y en el abordaje, en atención a su carácter dinámico, según las condiciones sociales, económicas y personales, que determinan la posibilidad de inserción en el mercado laboral (10).

Frente a las condiciones laborales de los odontólogos, uno de los indicadores relevantes como referente para el análisis de empleabilidad, es el de la proporción de graduados laborando en su profesión al momento de realización de la encuesta, que fue del $83 \%$. Este resultado es favorable si se analiza de manera independiente 
de otras variables, como el tipo de contrato, el promedio salarial, las garantías laborales, entre otras. En esta línea, los hallazgos muestran que la mayor parte de los odontólogos que afirmaron estar ejerciendo, lo hacían como profesionales independientes, seguidos de los que se encontraban empleados. Sin embargo, el 32\% de ellos contaba con contrato por prestación de servicios en distintas modalidades. Además, eran profesionales con fechas de graduación más reciente. El promedio salarial, en mayor medida, fue menor a \$1.500.000 mensuales (28,8\%). Teniendo en cuenta las limitaciones para hacer comparaciones por aspectos metodológicos como el tamaño muestral, el resultado es preocupante, por tratarse de un promedio de ingresos menor que el reportado por el Observatorio de Talento Humano en Salud para odontólogos en el 2017, que fue de $\$ 1.881 .470$ pesos mensuales. Adicionalmente, según este reporte, los odontólogos perciben ingresos más bajos que otros profesionales de la salud, como enfermeras, médicos e instrumentadores quirúrgicos (11). Para refrendar esta información, el estudio de seguimiento de egresados de programas académicos de pregrado realizado por la Pontificia Universidad Javeriana, que incluyó odontólogos, concluye que, con mayor frecuencia, los trabajadores con contrato de prestación de servicios perciben ingresos entre 500.000 y 1.500 .000 pesos (12).

En relación con los ingresos, también es importante resaltar, sin ser concluyentes por las razones anteriormente mencionadas, que en esta investigación se puso en evidencia la persistencia de la brecha en los ingresos entre hombres y mujeres. Las mujeres graduadas entre los años 2011 y 2016 están más cerca de los bajos ingresos, ubicándose en el rango de menos de 1.500.000 a 2.000.000, provenientes del ejercicio independiente de la profesión. Por el contrario, los hombres se ubican, en mayor medida, en la modalidad de empleados y perciben salarios entre $\$ 2.000 .000$ y $\$ 4.000 .000$. Este comportamiento desigual, según estudios del Observatorio Laboral del Ministerio de Educación de Colombia, es común a todas las áreas de conocimiento, siendo en el área de la salud en la que se presenta la mayor brecha (13).

Otro aspecto relevante, es el promedio de graduados que no ejercía la profesión al momento de la encuesta (16.9\%). De este total, aunque gran parte llevaba menos de seis meses sin trabajar como odontólogos (36,1\%), llama la atención que el 18\% no ejercía la profesión odontológica hacía más de dos años. Este tiempo es muy prolongado sin ejercer, máxime si se tiene en cuenta que el desarrollo continuo de las capacidades profesionales, requiere de su ejercicio permanente. Cuando el desempleo se prolonga por períodos superiores a un año acarrea consecuencias que van más allá de la falta de un ingreso, afectándose también los vínculos y corriendo el riesgo de experimentar una completa exclusión del mercado laboral (14). El promedio de odontólogos que nunca había ejercido su profesión al momento de la 
encuesta fue de 4.3\%. Esta cifra, se relaciona con la presentada por Llano y colaboradores, quienes reportan un 5\% de graduados que no se desempeñaba en la práctica odontológica (15).

El hecho de no ejercer se debe a razones como el no cumplimiento de expectativas de las ofertas del mercado laboral, y así lo afirmó el 39,4\% de los encuestados. La insatisfacción con las ofertas, tiene que ver con varios aspectos, entre los que se destaca el bajo salario ofrecido a los profesionales del nivel universitario y a la baja estabilidad laboral por el tipo de contratación (16). En correspondencia con esta afirmación, el 41,1\% de los graduados que estaban empleados, expresaron tener baja o muy baja satisfacción con el tipo de contratación, principalmente por la inestabilidad laboral.

Estos indicadores se relacionan, tanto en Colombia como en otros países de América Latina, con los nuevos escenarios laborales en los que se encuentran los odontólogos. Este tema es complejo, porque se debe -en parte- a la gran oferta universitaria para estudiar odontología, lo que llevó a que en el mediano plazo se elevara también el número de graduados (17). Así mismo, se relaciona con la escasa apertura de nuevos cupos laborales, aspecto que ha afectado más a los odontólogos que a otros profesionales de la salud. También la concentración de profesionales en las grandes ciudades ha influido en el deterioro de las condiciones laborales. Todas las situaciones mencionadas han derivado en la aparición de las primeras generaciones de odontólogos desempleados, o contratados bajo algunas de las modalidades de prestación de servicios en clínicas privadas dependientes de la demanda (18). Frente a esta problemática, es importante destacar que el desempleo juvenil y las condiciones laborales deficientes, son fenómenos estructurales del sistema productivo y de los desajustes existentes entre la formación y el mercado laboral (19), y aunque hay profesiones más afectadas que otras, como en el caso de la odontología, es una realidad común para gran parte de las profesiones y, por lo tanto, de los jóvenes del nivel universitario. En Colombia, según cifras del Dane a 2018, 3.400.000 jóvenes (entre 18 y 28 años) no tienen empleo (20).

Tanto el desempleo como las condiciones laborales precarias, generan afectaciones en distintos aspectos de las condiciones de vida. Entre ellos, está la cobertura en materia de seguridad social integral, dado que 60,3\% de los empleados por contrato indefinido o a término fijo, están totalmente cubiertos, mientras que en los independientes el porcentaje fue del 39,7\%, presentándose diferencias estadísticamente significativas por sexo a favor de los hombres. De los participantes que afirmaron contar con cobertura parcial o no contar con ninguna, el 84,5\% laboraban como independientes. En concordancia con lo expuesto, otros hallazgos investigativos han 
mostrado bajas coberturas en el Sistema de Seguridad Social Integral en trabajadores independientes, lo que genera gran preocupación por sus implicaciones en el detrimento de las condiciones de vida, pero más aún por la desprotección que pueden tener a futuro en cobertura en salud y en materia pensional.

La concentración de odontólogos en las grandes ciudades, se destaca como otro de los elementos importantes y, en este sentido, el presente estudio mostró que el 58,3\% de los participantes, residía y ejercía la profesión en Medellín. El Observatorio de Talento Humano en Salud confirma esta problemática, al reportar que para 2016, de un estimado de 47.500 odontólogos (incluyendo especialistas) en Colombia, el 70\% se concentró en seis departamentos, con mayor densidad en Bogotá (21/10000) y menor en Chocó (1,7/10000) (9).

Los graduados también evaluaron la formación otorgada por su Universidad. En este tópico, un 76\% manifestó haber recibido una formación adecuada, que les ha permitido afrontar los retos propios del mundo laboral. Este hallazgo fue similar al encontrado en la investigación realizada en la Universidad del Valle, en que el 83\% de los encuestados se encontraba de acuerdo con su proceso formativo (21). De igual manera, el estudio realizado por Yepes y colaboradores (22) muestra que la mayor parte de los graduados del programa afirman que se les brindó una excelente formación académica.

En relación con la formación académica, es relevante tener en cuenta que en este estudio, el $74,71 \%$ de los graduados se desempeñaba en la atención clínica, y solo el 1,18\% en investigación. Algunos de los estudios de seguimiento realizados en Colombia muestran que la mayor parte de los graduados se dedican a la práctica clínica (23) (24) (25). Estos resultados permiten de forma indirecta hacer la relación con el predominio de un énfasis curricular clínico, que puede también influir en las condiciones laborales, debido a la carencia de un perfil profesional más amplio, que posibilite diversificación del desempeño laboral del odontólogo.

\section{Conclusiones y recomendaciones}

- Algunos aspectos de la empleabilidad, que se exploran en el estudio, muestran resultados desfavorables, por la presencia de formas precarias de contratación, deterioro en los niveles de ingresos y dificultad para insertarse en el mercado laboral, en especial para los graduados de años más recientes. 
- El no cumplimiento de las expectativas de las ofertas laborales, es una de las razones para que un buen número de graduados no estuviera ejerciendo la profesión al momento de realizar el estudio.

- El tiempo de espera es alto tanto para el primer empleo, como para ubicarse laboralmente. Esta situación reduce las posibilidades de insertarse en el mercado laboral en algún momento, por la pérdida de competencias y de vínculos profesionales.

- Gran parte de los profesionales independientes están cubiertos parcialmente o no están cubiertos por el Sistema de Seguridad Social Integral y esta es una realidad que puede incidir en el deterioro de sus condiciones de vida.

- La prestación de servicios de atención clínica, continúa siendo la modalidad dominante de ejercicio profesional. Este hallazgo invita a pensar en la necesidad de contemplar acciones curriculares, con el fin de materializar el perfil del graduado que se declara en los lineamientos que orientan el quehacer de los programas de pregrado en las dos facultades de odontología.

- Es necesario trabajar como gremio para plantear alternativas que busquen el mejoramiento de la empleabilidad. El trabajo conjunto debe acoger el concepto amplio de empleabilidad y, desde esta perspectiva, abordar aspectos propios del mercado laboral, pero también de los enfoques curriculares de los programas de pregrado.

\section{Referencias}

1. Lombana JE, Muñoz AE. Competitividad, educación y empleabilidad: consideraciones para el desarrollo de los países. Clío América, 2017; 11(22): 169-176. https://doi. org/10.21676/23897848.2437

2. Cala MF, Gutiérrez CH, Barragán C. y Valero P. Empleabilidad: una aproximación a la conceptualización. Cuadernos Hispanoamericanos de Psicología 2011; 11 (1): 7-18.

3. Suárez-Lantarón B. Empleabilidad: análisis del concepto. Revista de investigación en educación 2016; 14(1):67-84. Disponible en: http://reined.webs.uvigo.es/index.php/reined/article/ view/225

4. República de Colombia. Ley 100 de 1993 "Por la cual se crea el sistema de seguridad social integral y se dictan otras disposiciones". 
Empleabilidad de los graduados de programas de pregrado en odontología de la ciudad de MedellínColombia, 2017-2018

5. Ministerio de Salud y Protección Social. Política Nacional de Talento Humano en Salud. Dirección de Desarrollo de Talento Humano en Salud. Bogotá: Colombia; 2018.

6. República de Colombia. Ley 30 de diciembre 28 de 1992 por el cual se organiza el servicio público de la Educación Superior.

7. Salazar-Barragán D, Ternera-Pulido JH, Tovar-Valencia S, Atuesta MF, Barragán-Ávila M. Caracterización de la situación actual de la profesión de odontólogo en Colombia. Mesa sectorial salud centro de formación talento humano en salud Bogotá; 2008.

8. Otálvaro Castro GJ, Zuluaga Salazar SM, Jaramillo Delgado G, Ternera Pulido JH, Sandoval Valencia S. Políticas de salud bucal en Colombia. Tendencias y puntos críticos para la garantía del derecho a la salud. Univ Odontol. 2019 ene-jun;38(80). https://doi.org/10.11144/ Javeriana.uo38-80.psbc

9. Ministerio de Salud y Protección Social, Cendex. Los recursos humanos de la salud en Colombia Balance, Competencias y Prospectiva; 2007.

10. Álvarez Gavilanes JE, Romero Fernández A. La empleabilidad de graduados universitarios en el contexto latinoamericano. Realidades de Uniandes, Ecuador Atenas Universidad de Matanzas 2015; 4 (32): 01-15.

11. Ministerio de Salud y Protección Social de Colombia. Observatorio de Talento Humano en Salud. Disponible en: http://ontalentohumano.minsalud.gov.co/Paginas/Inicio.aspx

12. Pontificia Universidad Javeriana. Estudio de seguimiento de egresados de programas académicos de pregrado de la sede central. Editorial Pontificia Universidad Javeriana, Bogotá; 2012. Disponible en: https://www.javeriana.edu.co/documents/15838/273636/SPEGRE2012 V1.pdf/7ef592cf-da1d-4394-a134-b8a7ce8c3338

13. Ministerio deEducación deColombia.ObservatorioLaboralparalaEducaciónDisponibleen:https://www.mineducacion.gov.co/sistemasinfo/Sistemas-de-Informacion-en-Ed-Superior-es pecializados/212301:Observatorio-Laboral-para-la-Educacion

14. De La Hoz FJ, Quejada R, Yánez M. El desempleo juvenil: problema de efectos perpetuos. Revista Latinoamericana de Ciencias Sociales, Niñez y Juventud, 2012;10 (1): 427-439.

15. Llano E, Yepes FL, Beltrán M, Suárez LE, Miranda M, Saldarriaga A. Seguimiento de los egresados de la Facultad de Odontología de la Universidad de Antioquia desde 1941 hasta el 2011. Parte I: caracterización sociodemográfica. Rev Fac Odontol Univ Antioq 2014; 25(Supl): S51-S66. 
16. Florez-Acosta JH, Atehortúa- Becerra SC, Arenas-Mejía AC. Las condiciones laborales de los profesionales de la salud a partir de la Ley 100 de 1993: evolución y un estudio de caso para Medellín. Rev. Gerenc. Polit. Salud, Bogotá-Colombia 2009; 8 (16): 107-131.

17. Donatres Girald JA, Rojas Amador MA, Jiménez Barbosa WG. Calidad de vida de los odontólogos en Colombia. Revisión exploratoria de la literatura. Universitas Odontológica 2017, 36 (76). Disponible en: https://www.redalyc.org/comocitar.oa?id=231252657004

18. Monsalves, MJ. La odontología que vivimos y no queremos: una crítica al escenario actual. Rev Chil Salud Pública 2012; 16 (2): 241-246.

19. Moreno-Minguez A. La empleabilidad de los jóvenes en España: Explicando el elevado desempleo juvenil durante la recesión económica. Rev. Int. Investig. Cienc. Soc. 2015; 11 (1): 3-20. Disponible en: https://dialnet.unirioja.es/servlet/articulo?codigo=5156077

20. Departamento Nacional de Estadística (DANE). Disponible en: https://www.dane.gov.co/index.php/estadisticas.../mercado-laboral-de-la-juventud Consultado25/02/2019.

21. Pérez-Flórez Y, Soto-Llanos L, Celis-Sánchez M. Perfil del odontólogo egresado de la Universidad del Valle. Rev. estomatología 1999; 8 (1): 16-26.

22. Yepes FL, Llano E, Beltrán M, Suárez LE, Miranda M, Saldarriaga A. Seguimiento de los egresados de la Facultad de Odontología de la Universidad de Antioquia desde 1941 hasta el 2011. Parte III. Vínculo facultad-egresado e impacto social del programa. Rev Fac Odontol Univ Antioq 2014; 25(Supl): S80-S95.

23. Cristo P, Caballero R, Atanache R, Bautista W. Impacto económico y laboral del Sistema General de Seguridad Social en Salud en el ejercicio profesional de los Odontólogos egresados del colegio odontológico colombiano 1979-1991. Revista CES Odontología 2005; 18 (2).

24. Neyra-Agudelo E, Martínez-Gómez JE, Madrid-Cruz P, Osorio-Gallego A, Gallego-Gómez CL, Ochoa-Acosta, EM. Posicionamiento profesional y laboral de los egresados de la Universidad Cooperativa de Colombia, Facultad de Odontología, periodo 2005-II al 2008-II Rev Nal Odo UCC 2010; 6(10): 65-70.

25. Vélez JE, Oquendo C, Puello PA. Perfil del egresado de pregrado de la Facultad de Odontología del CES 2000- 2004. Rev CES Odont 2008; 21(1):27-32. 